\section{O5 DEUTSCHER RÖNTGENKONGRESS}

7. Gemeinsamer Kongress der DRG und ÖRG 28. - 31. Mai 2014 Congress Center Hamburg

\section{Radiologie ist Diagnose und Therapie}

KONGRESSPRÄSIDENTEN

Prof. Dr. med. Stefan Diederich, Marienhospital Düsseldorf Prof. Dr. med. Johannes Lammer, Medizinische Universität Wien

DAS KONVENTIONELLE RÖNTGENBILD THORAXRADIOLOGIE INKLUSIVE HERZ ONKOLOGISCHE RADIOLOGIE INTERVENTIONELLE RADIOLOGIE MTRA-PROGRAMM

Deutsche Röntgengesellschaft Gesellschaft für medizinische Radiologie e.V. Ernst-Reuter-Platz 10 | D-10587 Berlin

ÖRG - Österreichische Röntgengesellschaft FiG Gesellschaft für Medizinische Radiologie und Nuklearmedizin Neutorgasse 9/2 a | AT-1010 Wien

WWW.ROENTGENKONGRESS.DE
Die Österreichische Röntgengesellschaft bedankt sich herzlich bei allen TeilnehmerInnen Sponsoren, Ausstellern und MitarbeiterInnen für die Unterstützung des OERG 2013 Kongress.

2014 würden wir uns freuen, wenn Sie auch am gemeinsamen Kongress der DRG und ÖRG in Hamburg wieder teilnehmen. 